\title{
Highly efficient ketone body treatment in multiple acyl-CoA dehydrogenase deficiency-related leukodystrophy
}

\author{
Matthias Gautschi ${ }^{1,2}$, Christian Weisstanner ${ }^{3}$, Johannes Slotboom ${ }^{3}$, Esmeralda Nava' ${ }^{1}$, Theres Zürcher ${ }^{1}$ and Jean-Marc Nuoffer ${ }^{1,2}$
}

BACKGROUND: Multiple acyl-CoA dehydrogenase deficiency- (MADD-), also called glutaric aciduria type 2, associated leukodystrophy may be severe and progressive despite conventional treatment with protein- and fat-restricted diet, carnitine, riboflavin, and coenzyme Q10. Administration of ketone bodies was described as a promising adjunct, but has only been documented once.

METHODS: We describe a Portuguese boy of consanguineous parents who developed progressive muscle weakness at 2.5 y of age, followed by severe metabolic decompensation with hypoglycaemia and coma triggered by a viral infection. Magnetic resonance (MR) imaging showed diffuse leukodystrophy. MADD was diagnosed by biochemical and molecular analyses. Clinical deterioration continued despite conventional treatment. Enteral sodium D,L-3-hydroxybutyrate (NaHB) was progressively introduced and maintained at $600 \mathrm{mg} / \mathrm{kg}$ BW/d $(\approx 3 \%$ caloric need). Follow up was 3 y and included regular clinical examinations, biochemical studies, and imaging.

RESULTS: During follow up, the initial GMFC-MLD (motor function classification system, $0=$ normal, $6=$ maximum impairment) level of 5-6 gradually improved to 1 after 5 mo. Social functioning and quality of life recovered remarkably. We found considerable improvement of MR imaging and spectroscopy during follow up, with a certain lag behind clinical recovery. There was some persistent residual developmental delay.

CONCLUSION: NaHB is a highly effective and safe treatment that needs further controlled studies.

M ultiple acyl-CoA dehydrogenase deficiency (MADD), also called glutaric aciduria type II (OMIM \#231680) is a genetic defect of the riboflavin-dependent mitochondrial electron transfer system (1). The defect is due to autosomal recessively transmitted mutations in either the electron transfer flavoprotein (ETF) dehydrogenase (ETF:Ubiquinone Oxidoreductase, ETFQO, OMIM *231675; EC 1.5.5.1; ETFDH gene) the most frequent, or the ETF subunits $\alpha$ and $\beta$ (ETFA, OMIM *608053, and ETFB, OMIM *130410, ETFA and ETFB genes, respectively). This leads to a secondary functional deficiency of a number of ETF-dependent dehydrogenases and results in a combined disorder of both fatty acid oxidation and amino acid metabolism (especially lysine, tryptophan, and branched-chain amino acids).

There is a wide spectrum of clinical phenotypes, ranging from pre- and neonatal, rapidly fatal, forms (1) to later-onset forms manifesting from juvenile to adult age (2-5). It may be severe or even fatal at any age $(6,7)$. Clinical manifestations include hypoketotic hypoglycaemia, metabolic acidosis, cardiomyopathy, neurodevelopmental delay with leukodystrophy and myopathy. Their expression and severity may vary within the same family (2).

Apart from the few vitamin-B2-responsive forms $(8,9)$, treatment has proven very difficult (7). Conventional treatment, including protein- and fat-restricted, carbohydrate-rich diet, ribofavin, glycine, and L-carnitine, may be effective in mildly affected patients, but is most of the time disappointing (1).

A racemic mixture of sodium D,L-3-hydroxybutyrate (NaHB) is a very promising treatment option. It aims at replacing the deficient endogenous ketone body production, needed not only for energy supply, but possibly more so for the synthesis of complex cell and tissue components such as myelin in the central nervous system. Its use was first reported in an abstract by Bonham et al. (10), in MADD, and subsequently in other conditions, such as treatment-refractory hypoglycaemia due to hyperinsulinism (11) and severe cardiomyopathy in glycogenosis type III (12). In 2003, Van Hove et al. (13) published three patients with MADD, one with predominant leukodystrophy and two with cardiomyopathy who responded very well to treatment with NaHB. Since this report, its efficacy for treating MADD has not been confirmed in another publication. Here, we provide extensive documentation of a patient with MADD who presented at $2.5 \mathrm{y}$ of age with severe leukodystrophy and who has remarkably improved on NaHB treatment both clinically and on magnetic resonance (MR) imaging and spectroscopy.

\section{RESULTS}

\section{Clinical Follow Up}

The NaHB treatment was well tolerated.

Since its introduction, the patient has never had any recurrence of metabolic decompensation, and has been in good general health. During the study period, an obstructive sleep apnea syndrome occurred, due to tonsil hypertrophy and 


\section{Articles | Gautschi et al.}

treated surgically, as well as allergic asthma; both conditions considered unrelated to either MADD or NaHB treatment.

Within a month's treatment, motor function began visibly to improve, there was some head control, he could grasp an object and was just able to sit with support. After 2 mo, he could sit up and remain seated alone for some minutes, head control was now good, he could clap in his hands and just stand with support. At $3 \mathrm{mo}$, sitting straight for more than $10 \mathrm{~min}$ was possible, he was able to stand with little support and do a few steps. At 4 mo, he crawled around and moved his arms above his head. At $5 \mathrm{mo}$, he was able to stand alone and play. At 6 mo, he walked around with only slight support and at $7 \mathrm{mo}$, he was walking without support and climbing stairs holding the handrail (Figure 1).

Normalization of mood and behavior after a few weeks treatment was another striking observation, which improved quality of life for him and his family considerably (data not shown).

Speech production was more affected than comprehension probably due to his motor disability. Within 2 mo of treatment, he increased his vocabulary and began to form two-word sentences. Bilingual speech and cognition have continued to improve since, without however normalizing so far (Table 1).
Fine motor function has also gradually improved with receding spastic-dystonic and ataxic movements (Figure 2). Strabismus disappeared, and apart from mild hyperopia and astigmatism (no correction needed), vision is now normal.

After about 8 mo of $\mathrm{NaHB}$ therapy, the pace of recovery slowed down. At 2.5 y follow up, the patient started to run, which had been difficult because of lack of sustained strength. Jumping still proved difficult, even though, generally, coordination was remarkably better. At 3 y, catch up of development continued; he had better control of gross motor function with almost normal gait and alternating steps on stairs.

Table 1 Developmental follow up

\begin{tabular}{lrrrrr} 
Treatment duration $(\mathrm{mo})$ & Start & 2 & 12 & 29 & 34 \\
\hline Chronological age $(\mathrm{y} ; \mathrm{mo})$ & $3 ; 11$ & $4 ; 1$ & $4 ; 11$ & $6 ; 4$ & $6 ; 9$ \\
Developmental age speech $(\mathrm{y} ; \mathrm{mo})$ & $1 ; 4$ & $1 ; 8$ & $3 ; 2$ & $4 ; 6$ & \\
DQ (speech) & 34 & 41 & 64 & 71 & \\
SON-IQ 2.5-7 (non-verbal) & & & 55 & & 64
\end{tabular}

DQ, developmental quotient; SON-IQ 2.5-7, Snijders-Oomen non-verbal intelligence quotient, for ages $2.5-7 \mathrm{y}$.

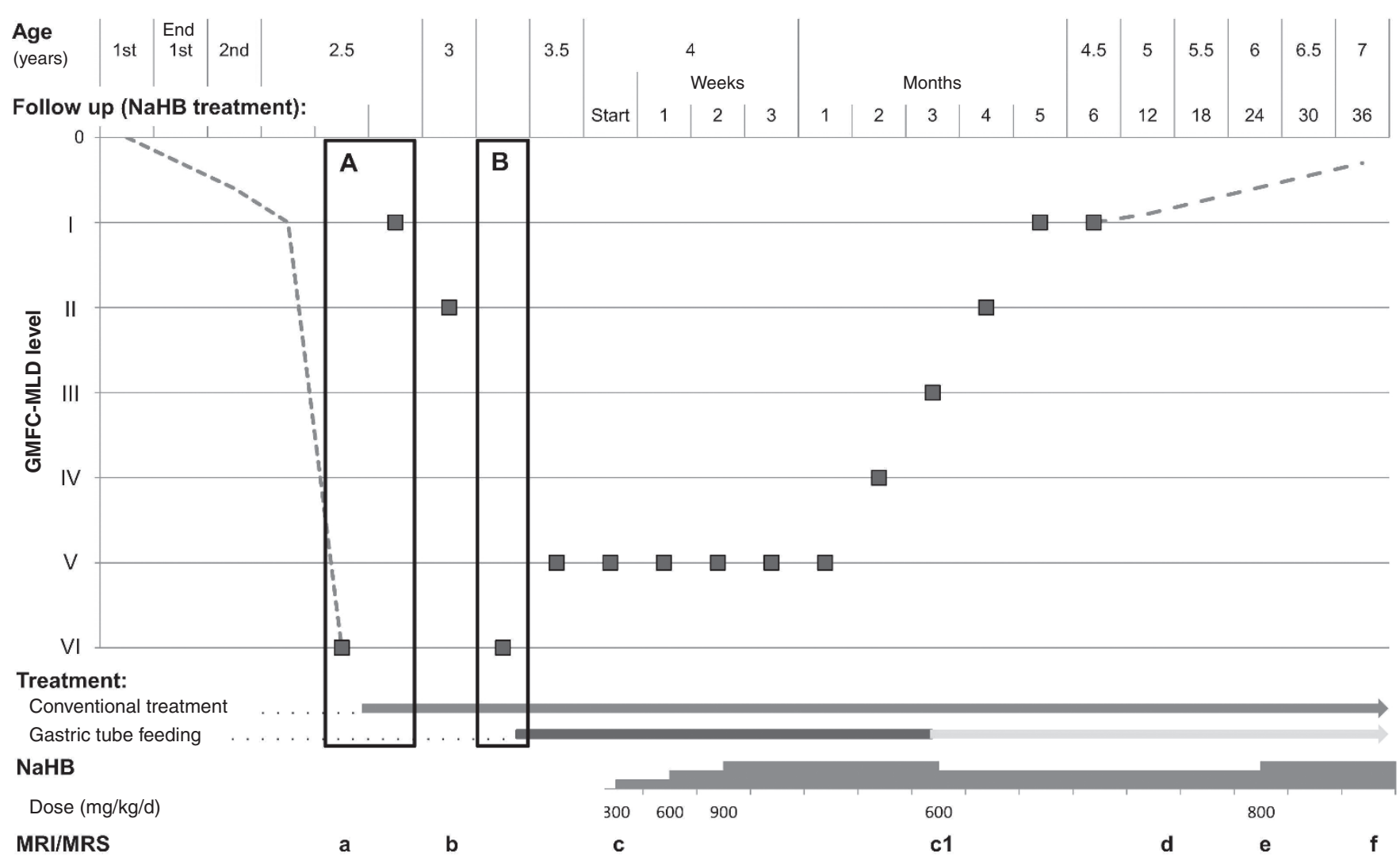

Figure 1 Treatment and follow-up scheme, and gross motor function. Schematic of the clinical course represented as gross motor function (Gross Motor Function Classification System developed for Metachromatic Leukodystrophy (GMFC-MLD) levels): retrospective estimation from patient file (dotted line); prospective assessment by clinical examination (squares). Continuing improvement, without reaching level 0 , i.e., without normalizing (dotted line after 6 mo of D,L-3-hydroxybutyrate (NaHB) treatment). Note that GMFC-MLD level is an ordinal system, not a score. Hospitalizations for metabolic decompensations are represented as black rectangles $\mathbf{A}$ and $\mathbf{B}$. After the 1st decompensation $(\mathbf{A})$, diagnosis was made and conventional treatment regimen with diet, riboflavin, and carnitine introduced. After the second decompensation (B): gastric tube feeding (dark gray: day and night, light gray: night only) and coenzyme Q10 substitution were started (see Methods). Magnetic resonance (MR) imaging and spectroscopy (a-f) were performed at the time points indicated. Time axis is not to scale. 


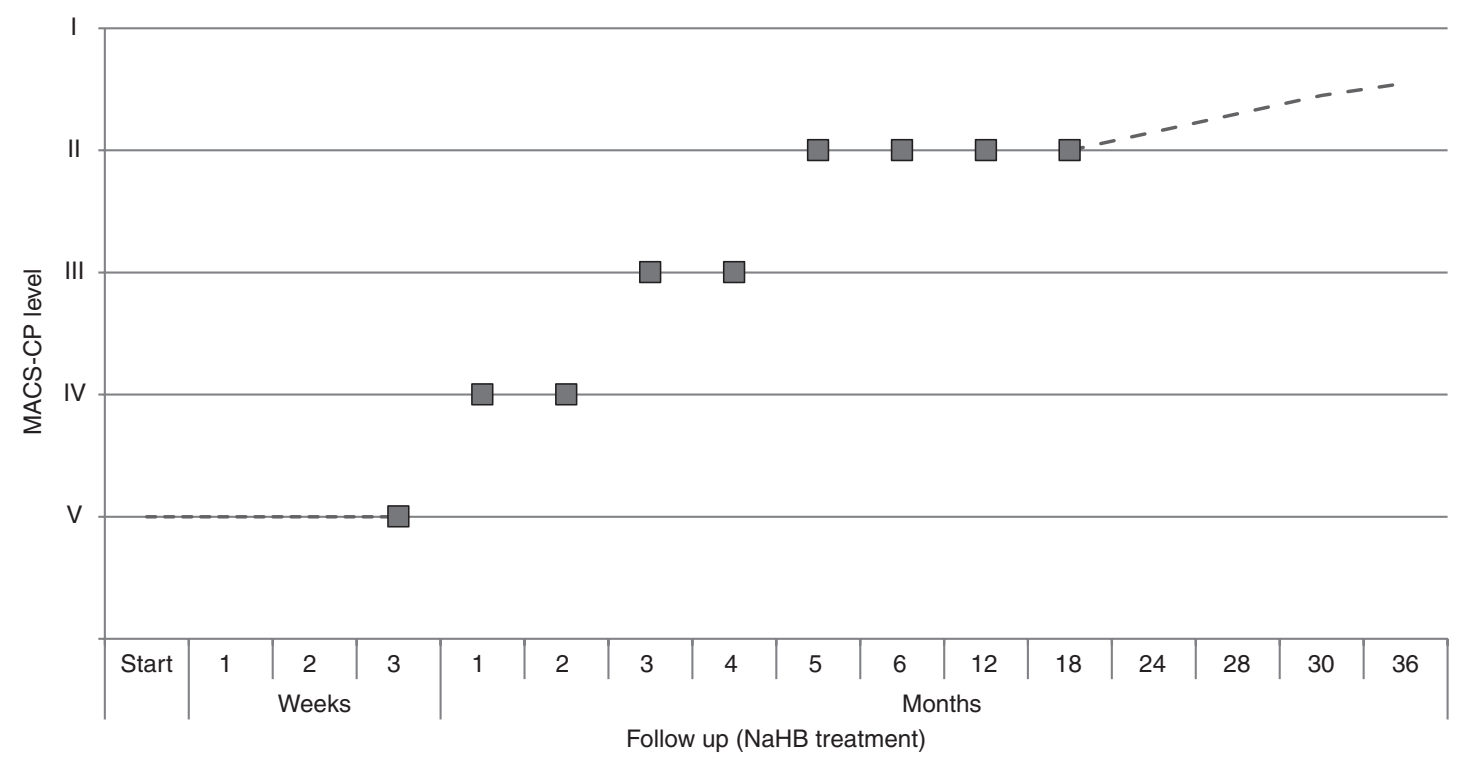

Figure 2 Manual ability level. Determined from occupational therapy assessments (squares) or estimated from patient file (dotted line). Note that the MACS is not a score but indicates a level of functioning. Manual ability continued to improve after 5 mo, however, without change of level. This is symbolized by the dotted upward line after 18 mo of treatment. The patient did not reach level I at 36 mo follow up. MACS-CP, Manual Ability Classification System for cerebral palsy; NaHB, sodium D,L-3-hydroxybutyrate.

\section{Biochemical Follow Up}

Without NaHB treatment and outside decompensation, plasma ketone bodies (i.e., acetoacetate (ACAC) and 3-hydroxybutyrate $(\mathrm{D} 3 \mathrm{HB}))$ were below the limit of detection $(<15 \mu \mathrm{mol} / \mathrm{l}$ and $<20 \mu \mathrm{mol} / \mathrm{l}$, respectively). After administration of a single dose of $\mathrm{NaHB}(150 \mathrm{mg} / \mathrm{kg})$, plasma ketone bodies increased to $123 \mu \mathrm{mol} / \mathrm{l}$ (ACAC $68 \mu \mathrm{mol} / \mathrm{l}$, D3HB $55 \mu \mathrm{mol} / \mathrm{l}$ ) within 1-2 h, i.e., in the normal range (reference range: $<200 \mu \mathrm{mol} / \mathrm{l}$, in the fed state, $1 \mathrm{~h}$ after meal (14)). Maximum plasma ketone bodies measured was $652 \mu \mathrm{mol} / \mathrm{l}$ (ACAC $309 \mu \mathrm{mol} / \mathrm{l}$, D3HB 343 $\mu \mathrm{mol} / \mathrm{l}) 2 \mathrm{~h}$ after a $200 \mathrm{mg} / \mathrm{kg} \mathrm{NaHB}$ loading dose.

We did not observe a systematic decrease in acylcarnitines, neither in response to a $\mathrm{NaHB}$ load, nor following chronic $\mathrm{NaHB}$ administration (data not shown).

Electrolytes and renal function remained normal throughout the follow-up period.

\section{MR Images}

MRI (Figure 3) was performed at the time points as indicated in Figure 1.

(a) At diagnosis, there were extensive symmetric demyelinating lesions of the white matter with slight frontal predominance, sparing the U-fibers, extensive swelling of the corpus callosum and mottled lesions also in the cerebellar hemispheres. All these areas showed restricted diffusion. Blood-brain barrier, as well as cortex and deep gray matter were intact.

(b) Six months later, progression with new diffusion restriction at the borders of known leukodystrophic areas, increased in the centrum semiovale, were observed. In T2-weighted images and FLAIR, globally increased and confluent lesions extending from the deep periventricular white matter to the subcortical white matter, still sparing the U-fibers.

(c) At the treatment start with $\mathrm{NaHB}, 16$ mo after diagnosis, there had been further progression of the extensive leukodystrophy including the cerebellum. Cortex and basal ganglia, as well as U-fibers and fiber tracts to the cerebral peduncles continued to be spared. Progressively narrow ventricles and sulci reflected increasing edema.

(c1) Three months after treatment start, slightly decreasing diffusion restriction of the periventricular white matter was seen (not shown in Figure 3).

(d) Fourteen months after treatment initiation, there was decreasing edema of the supra- and infratentorial white matter with regressing lesions of the cerebellum and regressing diffusion restriction of the periventricular white matter. Cerebral fluid space volume had normalized.

(e) At 24 mo of treatment, extension of leukodystrophy was unchanged compared to (d). On the other hand, a slight reduction of previously described subcortical diffusion restriction, and reduced swelling of corpus callosum were observed.

(f) After 3 y follow up, there was some further reduction of the diffusion restriction and of the leukodystrophic white matter including in the corpus callosum.

\section{MR Spectroscopy}

The follow up included MR spectroscopies (Figure 4), at the time points indicated in Figure 1. Follow up of ratios of the main spectroscopy peaks is summarized in Table 2.

(a) At initial diagnosis, the SVS spectroscopy of the frontal white matter showed elevated choline-to-creatine-ratio, elevated choline-to-NAA-ratio most likely due to 
a
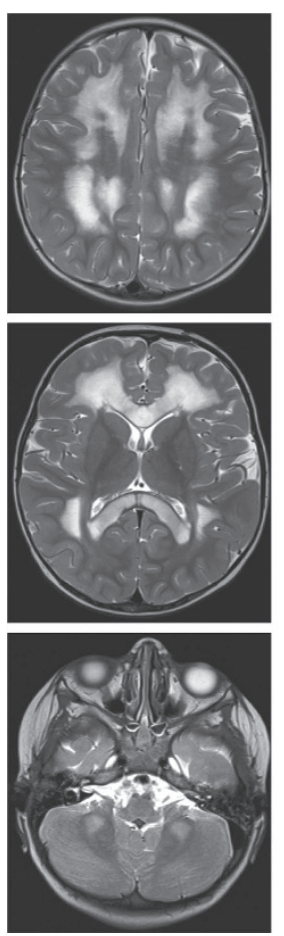

b
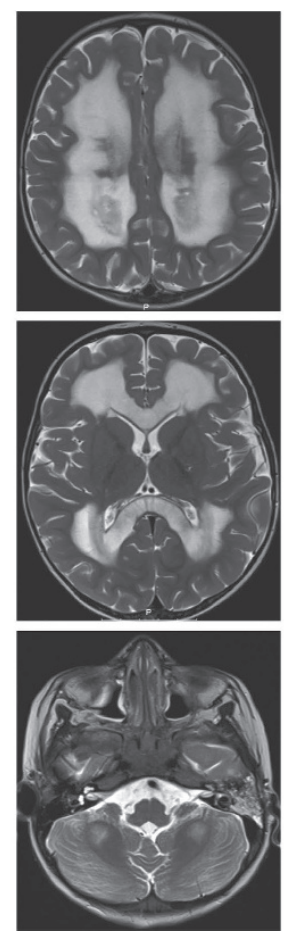

c
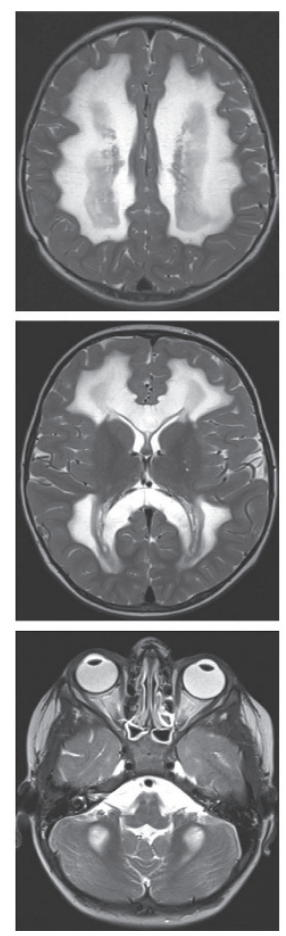

d
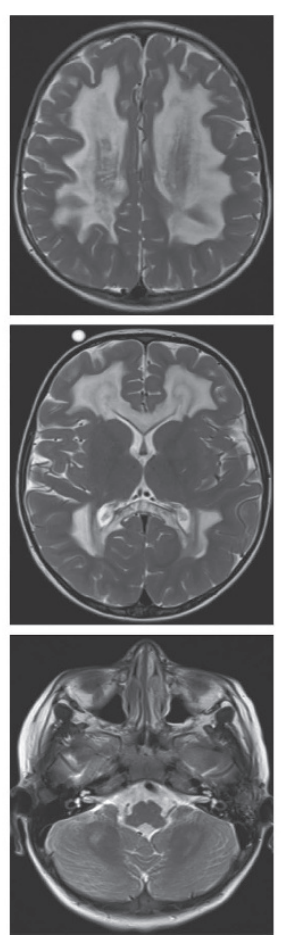

e
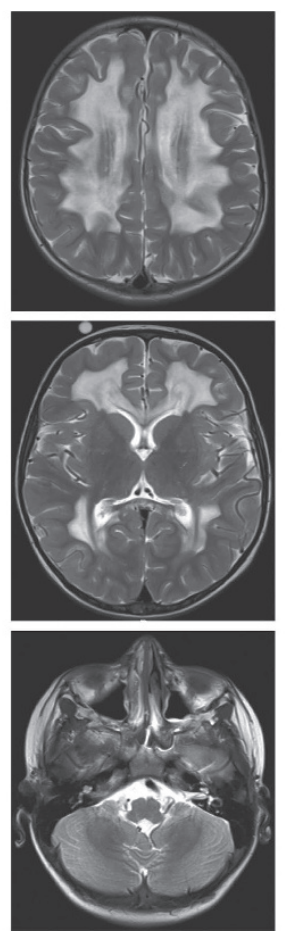

f
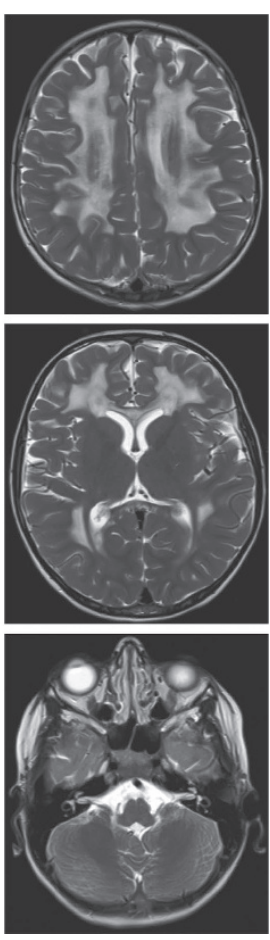

Figure 3 T2-weighted magnetic resonance (MR) images shown at three different levels: (i) directly above the corpus callosum (upper row), (ii) through the basal ganglia (middle row), and (iii) through the cerebellum (lower row), at six different time points (see Figure 1, from left to right): (a) MR imaging at diagnosis, (b) after 6 mo of conventional treatment, (c) at start of sodium D,L-3-hydroxybutyrate (NaHB) treatment, 16 mo after diagnosis, (d) after 14 mo, (e) after $24 \mathrm{mo}$, and (f) after 36 mo of NaHB treatment.

significantly reduced $\mathrm{N}$-acetylaspartate (NAA). The spectra of the patient showed massively elevated lactate. In the initial spectrum, the Lac-to-Cr-ratio was 0.88 . The short echo time spectra further showed slightly elevated signals which are attributed to mobile lipids: one broad resonance at $1.3 \mathrm{ppm}$ and one resonance at $0.9 \mathrm{ppm}$.

(b) Six months after diagnosis, there was a significant increase of the lactate peak both in the frontal white matter and the centrum semiovale, compared to the first MRI.

(c) At NaHB treatment start, there was still increased lactate, while NAA was markedly reduced, as well as choline and creatine, possibly due to the edema. The Cho/ Cr-ratio was also pathological.

(c1) After 3 mo, improving Lac/Cr-ratio but elevated Cho/Crand Cho/NAA-ratios, were seen, possibly due to increased membrane turnover in the cells (not shown in Figure 4).

(d) At $14 \mathrm{mo}$, substantial improvement of spectroscopy could be observed. While in the frontal white matter, there was still increased lactate and decreased NAA, lactate as well as choline-, creatine-, and NAA-ratios were markedly improved in the centrum semiovale. Choline distribution was normal and symmetrical, while NAA and creatine reduction was more pronounced in the frontal compared to the parieto-occipital regions. No macromolecule peaks were detected any more at 1.3 ppm. Spectroscopy of gray matter was also normal.

(e) At 24 mo after treatment initiation, spectroscopy of the frontal white matter was persistently abnormal, while it continued to improve at the level of the centrum semiovale, where the NAA had normalized. Two hours after an oral dose of $\mathrm{NaHB}(4 \mathrm{~g} \approx 180 \mathrm{mg} / \mathrm{kg})$ and in the short echo time (TE $=30 \mathrm{~ms})$, the lactate doublet was asymmetrical and showed a shoulder at its right side around $1.25 \mathrm{ppm}$, which may have been due to 3-hydroxybutyrate (Figure 4, arrow in MRS 6).

(f) At $36 \mathrm{mo}$, main metabolites and their ratios globally did not change significantly compared to the last spectroscopy. There was still reduced NAA. The highest $\mathrm{Cho} / \mathrm{Cr}$ ratios were in the frontal zones, and decreasing gradually from frontal to parietal. The CSI showed patchy distribution of lactate, no elevated mobile lipids indicating that cells were not apoptotic or necrotic but viable.

\section{DISCUSSION}

Some of the patients reported with MADD-related leukodystrophy were clearly responsive to riboflavin-treatment $(8,9)$. In contrast, our patient did not respond to several months of conventional treatment including riboflavin and there had been very little recovery 8 mo after the second decompensation. We therefore attribute the dramatic improvement after the introduction of NHB treatment largely to the action of ketone body supplementation.

The treatment was remarkably well tolerated and appeared safe during the whole follow-up period, with no side effects apart from some indigestion and tendency to dehydration in the beginning, 
a

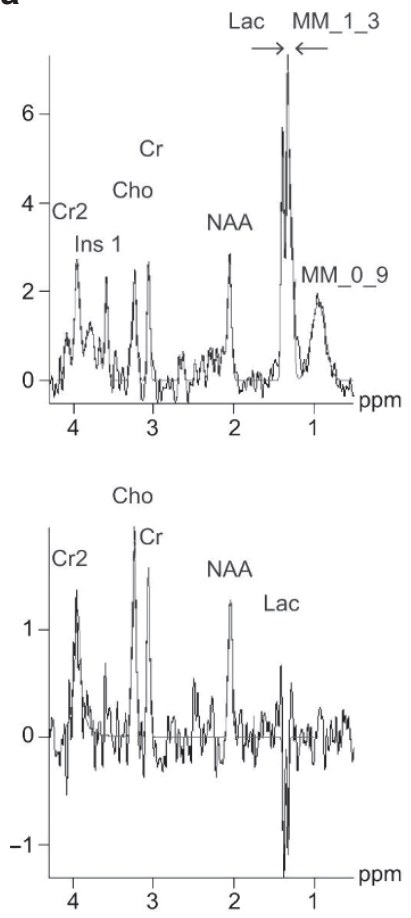

b
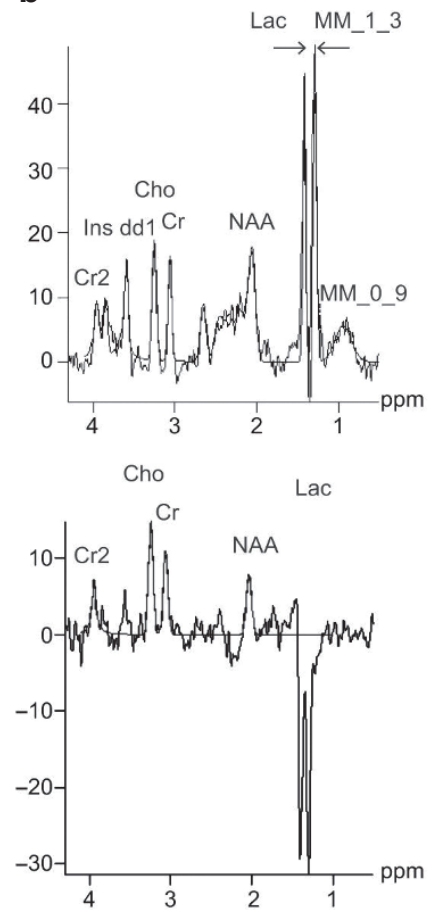

e
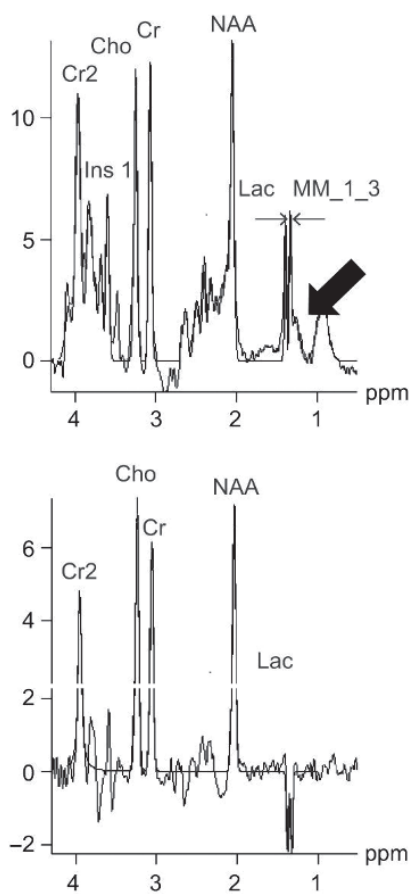

C
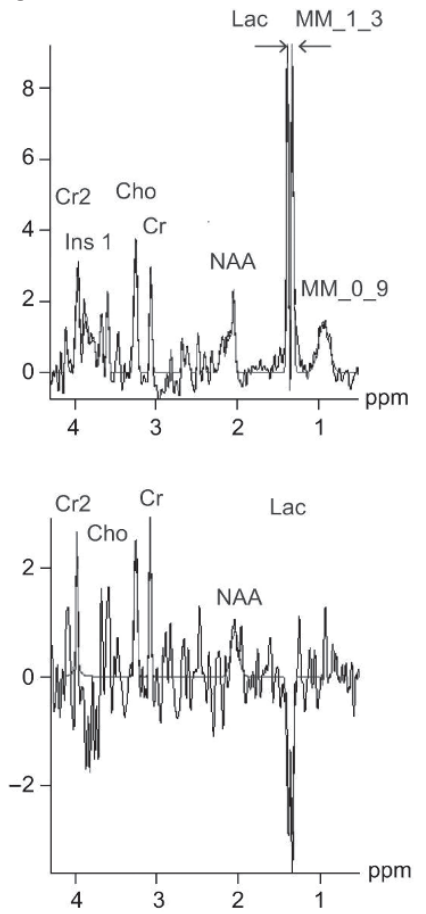

f

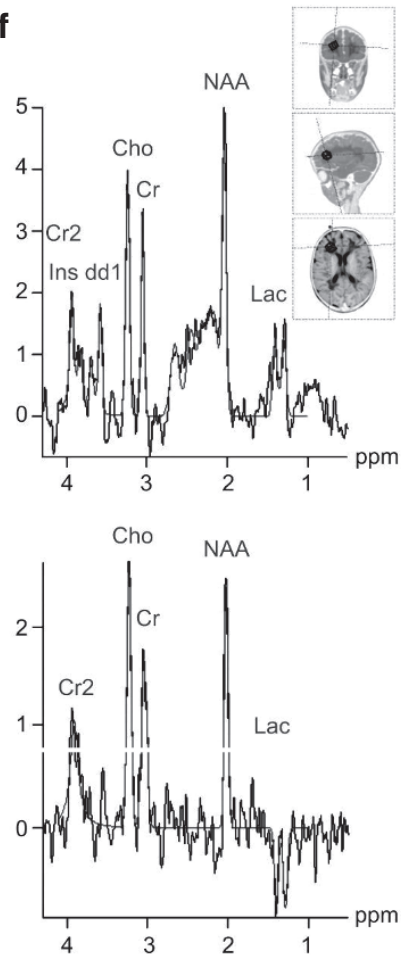

d
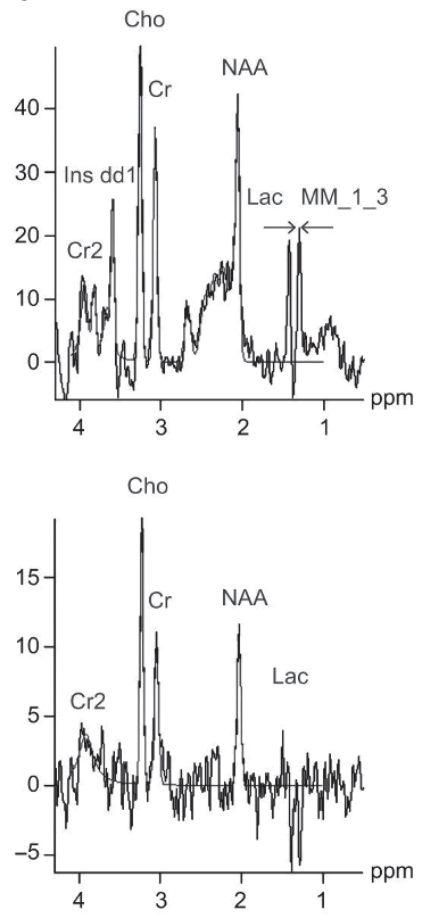

Figure 4 Follow up of magnetic resonance spectroscopy. Time points as indicated in Figure 1. All y axes have arbitrary units. (a-c) The lactate resonance at $1.33 \mathrm{ppm}$ is clearly visible in all spectra, but especially in spectra $\mathbf{a}-\mathbf{c}$. (d-f) The effect of the NaHB therapy can be appreciated in spectra d-f, where the reduction of the lactate with respect to the other resonances can clearly be viewed. The arrow in e points to the shoulder of the asymmetrical lactate doublet, possibly corresponding to a 3-hydroxybutyrate peak at $1.25 \mathrm{ppm}$. The insert in $\mathbf{f}$ shows the localization of the brain spectra.

which were treated with increased water intake. Renal function and electrolyte balance were stable despite the high salt load.

The maintenance dose of NaHB treatment (i.e., racemic mixture of D,L-3-hydroxybutyrate) corresponded to $3-4 \%$ of the daily caloric need. After NaHB administration, plasma ketone bodies (i.e., the sum of $\mathrm{ACAC}$ and $\mathrm{D} 3 \mathrm{HB}$ ) usually rose to normal non-fasting levels ( $<200 \mu \mathrm{mol} / \mathrm{l}, 1 \mathrm{~h}$ after meal). Their main action in the brain may therefore be the provision of building 
Table 2 Single voxel spectroscopy

\begin{tabular}{lcccc}
\hline & Cho/Cr-ratio & Cho/NAA & Lac/Cr & Field \\
\cline { 2 - 4 } MRS number & $\begin{array}{c}\text { Normal } \\
\text { value } \approx 1.10\end{array}$ & $\begin{array}{c}\text { Normal value } \\
\approx 0.63\end{array}$ & $\begin{array}{c}\text { Normal } \\
\text { value } \approx 0.0\end{array}$ & $\begin{array}{c}\text { strength } \\
\text { (Tesla) }\end{array}$ \\
\hline a & 1.52 & 1.43 & 0.88 & $1.5 \mathrm{~T}$ \\
$\mathrm{~b}$ & 1.54 & 1.50 & 5.26 & $3.0 \mathrm{~T}$ \\
$\mathrm{C}$ & 1.10 & 1.15 & 2.27 & $1.5 \mathrm{~T}$ \\
$\mathrm{C1}$ & 1.70 & 3.20 & 1.28 & $3.0 \mathrm{~T}$ \\
$\mathrm{~d}$ & 1.32 & 1.42 & 0.55 & $3.0 \mathrm{~T}$ \\
$\mathrm{e}$ & 1.23 & 1.07 & 0.47 & $1.5 \mathrm{~T}$ \\
$\mathrm{f}$ & 1.20 & 1.18 & 0.70 & $1.5 \mathrm{~T}$ \\
\hline
\end{tabular}

Observed routine spectroscopy ratios in the patients' (frontal/parietal) white matter. MRS letters correspond to time points shown in Figure 1.

Field strengths of MR imaging and spectroscopy instruments used (Siemens Magnetom instruments, Avanto for MRS no a, c, and e, Sonata for f and Verio for b, c1, and d).

blocks, especially for re-myelination rather than the potential energy supply. On the other hand, the effective amount of ketone bodies may have been underestimated, as 3-hydroxybutyrate was measured using an enzymatic test specific for the D-enantiomer, while the NaHB used for treatment was a D,Lracemic mixture. In humans, the $\mathrm{D}$-enantiomer is produced by ketogenesis in the liver and metabolized by extra-hepatic tissues including the brain (15). L-3-hydroxybutyryl-CoA is an intermediate metabolite of fatty acid $\beta$-oxidation, which can be converted to L-3-hydroxybutyrylcarnitine, but it is not a predominant metabolite even in ketosis (16). Little is known about the uptake and metabolization of free L-3-hydroxybutyrate by peripheral organs and its physiological importance in humans. In rats, it has been shown, that the L-enantiomer is produced by the liver (17) and can be utilized by the developing brain (18) and that there is a stereoselective effect of the enantiomers in cardiomyocytes (19). L-3-hydroxybutyrate may be thus converted to physiological ketone bodies, diverted to biosynthesis of lipids, sterols and other complex molecules or fully metabolized to CO2 by the Krebs cycle $(20,21)$. Because of the methods we used, we were not able to assess for uptake, kinetics and effect of the L-enantiomer in our case.

The clinical effect of oral ketone body treatment was very impressive. It became obvious within the first few weeks of treatment. The natural course of disease and recovery under treatment appear similar to that reported by Van Hove et al. (13). $\mathrm{NaHB}$ treatment appeared to effectively stabilize the patient as he did not experience any further decompensation despite several febrile infections and other situations at risk. In contrast, untreated riboflavin non-responsive MADD can lead to recurrent, even fatal, decompensations difficult to manage (22).

Neurological recovery in terms of gross and fine motor function was assessed using validated classification systems. These classifications are only a rough measure of motor functioning and do not render well the continuing but more subtle clinical recovery after 6-8 mo under NaHB treatment. However, despite the considerable improvement at all the clinically relevant levels, notably, of mood, behavior, gross and fine motor function, including ocular movements, speech and cognition, some degree of motor dysfunction and mental retardation persisted at 36 mo of follow up. Irreversible brain damage that occurred prior to treatment or brain functions that are not (or only partly) amenable to NaHB treatment cannot be excluded.

Our patient did not have hypertrophic cardiomyopathy, though it occurs frequently in the context of MADD (23) and was reported a prominent feature of fatal MADD in several infants $(7,24-26)$. It was shown to respond equally to $\mathrm{NaHB}$ treatment (13). In view of the effectiveness of the NaHB treatment, irreversible damage to the brain or fatal cardiomyopathy could potentially be avoided by including MADD in the newborn screening (NBS) programs.

While we observed similar blood ketone levels as Van Hove et al. (13) after a single NaHB dose, as well as a decrease in serum free fatty acids, we did not observe a systematic decrease in acylcarnitines, and therefore no clear correlation between clinical and biochemical improvement.

Liver, muscles and heart were only minimally affected in our patient. In contrast, liver and muscle enzymes are commonly elevated in MADD-patients (27). Liver involvement can present as Reye-like syndrome (3) and myopathy as muscular weakness, including respiratory insufficiency (22) and rhabdomyolysis (6).

Our case illustrates the extensive leukodystrophy, including both cerebrum and cerebellum, that can occur in lateonset MADD. Only a few patients have been well documented $(8,9,13,23)$. Sometimes, the deep gray matter is also involved (28). We did not observe this in our case, even though the nature of our patient's motor disorder with marked dystonia points to basal ganglia dysfunction.

Interestingly, improvement of the MR images lagged behind clinical recovery. The predominant white matter abnormalities receded only very slowly, and within the $3 \mathrm{y}$ of follow up, only little, even though consistently. In contrast to the previous report (13), we did not observe the formation of white matter cavitation in our patient.

On MR spectroscopy, the $\mathrm{Lac} / \mathrm{Cr}$ ratio showed continuous improvement after start of the NaHB treatment and may be a marker for the severity of the disease. However, while there is some correlation between severity of clinical manifestations and increased $\mathrm{Lac} / \mathrm{Cr}$ ratio, their relationship is not a simple one.

\section{Conclusions}

This single-case study shows the impressive clinical and radiological effectiveness of D,L-3-hydroxybutyrate for the treatment of severe MADD-related leukodystrophy. It appeared to be safe and well tolerated over the study period of $3 \mathrm{y}$. There was rapid clinical improvement during the first 6-8 mo, followed by a continued albeit slower pace of catch-up development. Brain imaging and spectroscopy improved with a certain time lag compared to the recovery of motor function. More and larger controlled studies are needed to show the full potential and limitation of this treatment including in patients diagnosed by newborn screening. 


\section{METHODS}

\section{Case Report}

This study was performed in compliance with the local ethics board directives, as well as with the Helsinki Declaration (1975, rev. 2000). Written informed consent for both the experimental treatment and the publication of the case were obtained from the parents.

The disease course is summarized in Figure 1.

The 2.5-y-old boy of consanguineous Portuguese parents was brought to the emergency department in a comatose state, in the context of a mild viral infection and recurrent vomiting. Prior to this acute decompensation he had developed a progressive muscular weakness over the last 6 mo.

At initial work up, he had severe hypoglycaemia and carnitine deficiency, with mildly increased liver enzymes, but normal creatine kinase and lactate.

Plasma acylcarnitines and urinary organic acids were suggestive of MADD. A novel homozygous mutation in Exon 9 (c.1106G $>$ C, p.Gly369Ala) in the ETFDH gene confirmed the diagnosis. In silico simulation indicated its "probably damaging" nature (PolyPhen-2 (v2.2.2r398) score 1.000; (29)).

Further work-up showed ultrasonographic signs of liver steatosis, normal kidneys and heart.

At hospital discharge, there was a mild psychomotor retardation, especially of language (developmental quotient 80 ). He had a squint and suspicion of reduced vision, a mixed spastic-dystonic motor disability with ataxic components, weak head control, a walking distance of 10 meters, and marked mood instability with frequent crying spells.

A second metabolic decompensation occurred 8 mo later, again triggered by a viral infection. Although biochemically less pronounced, it left the patient with a severe spastic-dystonic tetraparesis and bedridden. He was unable to lift his head and to swallow his saliva. He was also strikingly unhappy and crying most of the time. A gastrostomy was placed in order to simplify the administration of his diet.

At 8 mo after the second decompensation, the boy had regained a vocabulary of four to five single words, but had otherwise made only very little recovery and remained completely bed-ridden. NaHB therapy was started, as described below.

\section{Treatment}

The treatment scheme is summarized in Figure 1.

Conventional treatment. A fat- and protein-restricted diet was introduced after diagnosis. Gastric tube feeding was maintained as needed to prevent catabolism. He received carnitine, riboflavin $(100 \mathrm{mg} / \mathrm{d})$, and ubiquinone (5-10 $\mathrm{mg} / \mathrm{kg} \mathrm{BW/d})$, after idebenone had been given for 6 mo following the second decompensation. The patient also received oral baclofen $(5 \mathrm{mg} / \mathrm{d})$ to relieve his dystonia.

NaHB treatment. Racemic mixture of sodium D,L-3-hydroxybutyrate (NaHB; M2i, Salin-de-Giraud, France) was produced by the hospital pharmacy (recipients of 1,2 , or $2.5 \mathrm{~g}$ ).

Enteral $\mathrm{NaHB}$ was administered by gastrostomy every $4 \mathrm{~h}$ at $300 \mathrm{mg} / \mathrm{kg} \mathrm{BW} / \mathrm{d}$, progressively increased to $900 \mathrm{mg} / \mathrm{kg} \mathrm{BW} / \mathrm{d}$ and, after $3 \mathrm{mo}$, maintained at $600 \mathrm{mg} / \mathrm{kg} \mathrm{BW} / \mathrm{d}(\approx 3 \%$ caloric need). After follow up of $24 \mathrm{mo}$, the dose was increased to three hourly administration of the same single dose to a daily dose of $\sim 800 \mathrm{mg} / \mathrm{kg} \mathrm{BW} / \mathrm{d}$, because of the boy's growth, as well as apparent slowing of clinical and neuroradiological recovery (see below).

\section{Clinical Follow Up}

Follow up was done every week for the first month, every month for the first 6 mo, and every 6 mo thereafter.

Clinical monitoring included regular general physical and thorough neurological examinations at every follow-up visit. This assessment was summarized using the Gross Motor Function Classification System developed for Metachromatic Leukodystrophy (GMFCMLD) (30). Briefly, level 0 corresponds to walking with normal quality for age, level I to walking without support but with reduced quality of performance (i.e., instability). Level II is defined by walking with support, and up to five free steps, whereas level III means no walking at all, while some locomotion (e.g., crawling) as well as sitting without support is still possible. At level IV, sitting without support is still possible, but no locomotion; at level V no locomotion or sitting without support is possible but head control is preserved. Finally, level VI means loss of any locomotion, no head and no trunk control. In this classification system, level 0 was introduced to define a potentially normal functional outcome of treatment.

Global bi-manual function was classified using the Manual Ability Classification System for cerebral palsy (MACS-CP) (31). No level 0 was defined in this classification system. Level I means that the patient handles objects easily and successfully. Level II corresponds to handling of objects with somewhat reduced quality and/or speed, while at level III, objects are handled with difficulty. Level IV is defined as handling a limited selection of easily managed objects in adapted situations. At level V, the patient does not handle objects, and has a severely limited ability to perform even simple actions. MACS-CP level was determined retrospectively from regular occupational therapy assessments.

Neuropsychological testing included language-independent cognitive testing (SON-R 2.5-7; Snijders-Oomen non-verbal intelligence test, revised, for ages $2.5-7$ years) as well as assessment of speech development, using several tests depending on age and ability of the patient. The tests were performed by a neuropsychologist and a speech therapist, respectively.

In addition, the progress of the patient was documented with videos taken by the physiotherapist at regular intervals, and with a quality of life questionnaire, which was filled out by the parents at every follow-up visit for $1 \mathrm{y}$.

\section{MR Imaging and Spectroscopy}

For reason of availability, several different instruments were used for the MR imaging and spectroscopy (Magnetom Avanto 1.5T, Sonata 1.5T and Verio 3.0T; Siemens, Erlangen, Germany); examinations a, $\mathrm{c}$, and e were performed at 3.0 Tesla, examinations $\mathrm{b}, \mathrm{cl}$, $\mathrm{d}$, and $\mathrm{f}$ at 1.5 Tesla (Table 2).

MR imaging. MR imaging consisted of diffusion imaging, T2, Flair, $\mathrm{T} 1$ at each time point. At three examination dates gadolinium was given intravenously.

MR spectroscopy. The MR spectroscopy (MRS) consisted of chemical shift imaging (CSI-PRESS) at an echo time TE of $30 \mathrm{~ms}$ enabling the detection of mobile fatty acids, and at TE of $135 \mathrm{~ms}$, for the detection of lactate, which shows up as an inverted doublet at $1.33 \mathrm{ppm}$ (see Figure 4). Additionally, two single voxel spectroscopy (SVS-) PRESS measurements have been carried out according to a protocol described by (32) for quantitative MRS. The standard spectroscopy post processing protocols of Siemens were used for qualitative MRSanalysis. The metabolites examined were choline (Cho), creatine $(\mathrm{Cr})$, $\mathrm{N}$-acetyl aspartate (NAA), lactate (Lac), and mobile lipids (ML).

\section{Biochemical Analyses}

Plasma acylcarnitine was performed with an LC-MS-MS System (API 4000 Q-Trap) and amino acids on a aminoacid analyzer (Biochrom 30). Urinary organic acid analysis was done with GC-MS and plasma ketone bodies (i.e., acetoacetate (ACAC) and D-3-hydroxybutyrate $(\mathrm{D} 3 \mathrm{HB})$ ) were quantified spectrophotometrically using the D-3hydroxybutyrate-dehydrogenase method (33). This enzymatic method measures only the D-enantiomer of 3-hydroxybutyrate but not the L-form. The inter-assay coefficient of variation for D-3hydroxybutyrate was $<6 \%$. In the urinary organic acids, total excretion of D- and L-3-hydroxybutyrate is measured.

\section{ACKNOWLEDGMENTS}

We thank André Schaller for help with the interpretation of the genetic data, Mary Butz-Myrick for critical reading of the manuscript (English), and Silvan Schmidhalter for help in preparing the figures. We thank our young patient and his family for their great effort of collaboration. Part of this study was presented at the German APS meeting in Fulda, spring 2012 and previously published as an abstract for the SSIEM Symposium in Birmingham, September 2012. Mutation analysis of the ETF A\&B and ETFDH genes was done by the laboratory Medizinisch Genetisches Zentrum (MGZ), Munich, Germany.

Disclosure: The authors declare no conflict of interest. 


\section{REFERENCES}

1. Frerman F, Goodman SI. Defects of electron transfer flavoprotein and electron transfer flavoprotein-ubiquinone oxidoreductase: glutaric acidemia type II. In: Scriver CR, Beaudet AL, Sly WS, Valle D, eds. The Metabolic and Molecular Bases of Inherited Disease. 8th ed. New York: McGraw-Hill, 2001:2357-65.

2. Dusheiko G, Kew MC, Joffe BI, Lewin JR, Mantagos S, Tanaka K. Recurrent hypoglycemia associated with glutaric aciduria type II in an adult. N Engl J Med 1979;301:1405-9.

3. Bell RB, Brownell AK, Roe CR, et al. Electron transfer flavoprotein: ubiquinone oxidoreductase (ETF:QO) deficiency in an adult. Neurology 1990;40:1779-82.

4. Pollard LM, Williams NR, Espinoza L, et al. Diagnosis, treatment, and long-term outcomes of late-onset (type III) multiple acyl-CoA dehydrogenase deficiency. J Child Neurol 2010;25:954-60.

5. Wolfe LA, He M, Vockley J, et al. Novel ETF dehydrogenase mutations in a patient with mild glutaric aciduria type II and complex II-III deficiency in liver and muscle. J Inherit Metab Dis 2010;33:Suppl 3:S481-7.

6. Lee HC, Lai CK, Siu TS, et al. Role of postmortem genetic testing demonstrated in a case of glutaric aciduria type II. Diagn Mol Pathol 2010;19:184-6.

7. Niederwieser A, Steinmann B, Exner U, et al. Multiple acyl-Co A dehydrogenation deficiency (MADD) in a boy with nonketotic hypoglycemia, hepatomegaly, muscle hypotonia and cardiomyopathy. Detection of N-isovalerylglutamic acid and its monoamide. Helv Paediatr Acta 1983;38:9-26.

8. Ramos Fernandez JM, Martinez-Pardo M, Garcia Villanueva M, et al. Miopatia lipidica por aciduria glutarica tipo II sensible a riboflavina. An Esp Pediatr 1995;42:207-10.

9. Uziel G, Garavaglia B, Ciceri E, Moroni I, Rimoldi M. Riboflavin-responsive glutaric aciduria type II presenting as a leukodystrophy. Pediatr Neurol 1995;13:333-5.

10. Bonham JR, Tanner MS, Pollitt RJ, et al. Oral sodium 3-hydroxybutyrate, a novel adjunct to treatment for multiple acyl CoA dehydrogenase deficiency. J Inherit Metab Dis 1999;22:101.

11. Plecko B, Stoeckler-Ipsiroglu S, Schober E, et al. Oral beta-hydroxybutyrate supplementation in two patients with hyperinsulinemic hypoglycemia: monitoring of beta-hydroxybutyrate levels in blood and cerebrospinal fluid, and in the brain by in vivo magnetic resonance spectroscopy. Pediatr Res 2002;52:301-6.

12. Valayannopoulos V, Bajolle F, Arnoux JB, et al. Successful treatment of severe cardiomyopathy in glycogen storage disease type III With D,L-3-hydroxybutyrate, ketogenic and high-protein diet. Pediatr Res 2011;70:638-41.

13. Van Hove JL, Grünewald S, Jaeken J, et al. D,L-3-hydroxybutyrate treatment of multiple acyl-CoA dehydrogenase deficiency (MADD). Lancet 2003;361:1433-5.

14. Vassault A. Lactate, pyruvate, acetoacetate and 3-hydroxybutyrate. In: Blau N, Duran N, Gibson KM, eds. Laboratory Guide to the Methods in Biochemical Genetics. Berlin, Germany: Springer-Verlag, 2008:35-51.

15. Fukao T, Lopaschuk GD, Mitchell GA. Pathways and control of ketone body metabolism: on the fringe of lipid biochemistry. Prostaglandins Leukot Essent Fatty Acids 2004;70:243-51.
16. Soeters MR, Serlie MJ, Sauerwein HP, et al. Characterization of D-3-hydroxybutyrylcarnitine (ketocarnitine): an identified ketosis-induced metabolite. Metab Clin Exp 2012;61:966-73.

17. Reed WD, Ozand PT. Enzymes of L-(+)-3-hydroxybutyrate metabolism in the rat. Arch Biochem Biophys 1980;205:94-103.

18. Swiatek KR, Dombrowski GJ Jr, Chao KL. The metabolism of Dand L-3-hydroxybutyrate in developing rat brain. Biochem Med 1984;31:332-46.

19. Tsai YC, Chou YC, Wu AB, et al. Stereoselective effects of 3-hydroxybutyrate on glucose utilization of rat cardiomyocytes. Life Sci 2006;78:1385-91.

20. Lincoln BC, Des Rosiers C, Brunengraber H. Metabolism of S-3-hydroxybutyrate in the perfused rat liver. Arch Biochem Biophys 1987;259:149-56.

21. Edmond J. Ketone bodies as precursors of sterols and fatty acids in the developing rat. J Biol Chem 1974;249:72-80.

22. Olsen RK, Pourfarzam M, Morris AA, et al. Lipid-storage myopathy and respiratory insufficiency due to ETFQO mutations in a patient with lateonset multiple acyl-CoA dehydrogenation deficiency. J Inherit Metab Dis 2004;27:671-8.

23. al-Essa MA, Rashed MS, Bakheet SM, Patay ZJ, Ozand PT. Glutaric aciduria type II: observations in seven patients with neonatal- and late-onset disease. J Perinatol 2000;20:120-8.

24. Goodman SI, Stene DO, McCabe ER, et al. Glutaric acidemia type II: clinical, biochemical, and morphologic considerations. J Pediatr 1982;100:946-50.

25. Angle B, Burton BK. Risk of sudden death and acute life-threatening events in patients with glutaric acidemia type II. Mol Genet Metab 2008;93:36-9.

26. Singla M, Guzman G, Griffin AJ, Bharati S. Cardiomyopathy in multiple Acyl-CoA dehydrogenase deficiency: a clinico-pathological correlation and review of literature. Pediatr Cardiol 2008;29:446-51.

27. Tamaoki Y, Kimura M, Hasegawa Y, Iga M, Inoue M, Yamaguchi S. A survey of Japanese patients with mitochondrial fatty acid beta-oxidation and related disorders as detected from 1985 to 2000. Brain Dev 2002;24:675-80.

28. Ho VB, Fitz CR, Chuang SH, Geyer CA. Bilateral basal ganglia lesions: pediatric differential considerations. Radiographics 1993;13:269-92.

29. Adzhubei IA, Schmidt S, Peshkin L, et al. A method and server for predicting damaging missense mutations. Nat Methods 2010;7:248-9.

30. Kehrer C, Blumenstock G, Raabe C, Krägeloh-Mann I. Development and reliability of a classification system for gross motor function in children with metachromatic leucodystrophy. Dev Med Child Neurol 2011;53:156-60.

31. Eliasson AC, Krumlinde-Sundholm L, Rösblad B, et al. The Manual Ability Classification System (MACS) for children with cerebral palsy: scale development and evidence of validity and reliability. Dev Med Child Neurol 2006;48:549-54.

32. Scheidegger O, Wingeier K, Stefan D, et al. Optimized quantitative magnetic resonance spectroscopy for clinical routine. Magn Reson Med 2013;70:25-32.

33. Bergmeyer HU. 3-hydroxybutyrate dehydrogenase. In: Bergmeyer HU, Bergmeyer J, Grassl M, eds. Methods of Enzymatic Analysis. 3rd ed. Weinheim, Germany: Verlag Chemie, 1983:226-8. 